\title{
On the Identification of the Large-Scale Properties of Tropical Convection Using Cloud Regimes
}

\author{
JACKSON TAN AND CHRISTIAN JAKOB \\ ARC Centre of Excellence for Climate System Science, Monash University, Melbourne, Australia \\ TODD P. LANE \\ School of Earth Science and ARC Centre of Excellence for Climate System Science, The University of Melbourne, \\ Melbourne, Australia
}

(Manuscript received 17 August 2012, in final form 5 February 2013)

\begin{abstract}
The use of cloud regimes in identifying tropical convection and the associated large-scale atmospheric properties is investigated. The regimes are derived by applying cluster analysis to satellite retrievals of daytime-averaged frequency distributions of cloud-top pressure and optical thickness within grids of $280 \mathrm{~km}$ by $280 \mathrm{~km}$ resolution from the International Satellite Cloud Climatology Project between 1983 and 2008. An investigation of atmospheric state variables as a function of cloud regime reveals that the regimes are useful indicators of the archetypal states of the tropical atmosphere ranging from a strongly convecting regime with large stratiform cloudiness to strongly suppressed conditions showing a large coverage with stratocumulus clouds. The convectively active regimes are shown to be moist and unstable with large-scale ascending motion, while convectively suppressed regimes are dry and stable with large-scale descending winds. Importantly, the cloud regimes also represent several transitional states. In particular, the cloud regime approach allows for the identification of the "building blocks" of tropical convection, namely, the regimes dominated by stratiform, deep, and congestus convection. The availability of the daily distribution of these building blocks for more than 20 years opens new avenues for the diagnosis of convective behavior as well as the evaluation of the representation of convection in global and regional models.
\end{abstract}

\section{Introduction}

Convection in the tropics is a key driver of the global atmospheric circulation and plays a major role in Earth's weather and climate. Being the primary mechanism for the transport of heat, momentum, and moisture, not only from the local surface to the troposphere but also laterally through planetary circulations such as the Hadley cells, its effect manifests on many scales. Among the important meteorological phenomena that it governs are the formation of clouds, the regional distribution of precipitation, and intraseasonal variations such as the Madden-Julian oscillation.

Despite its importance, the representation of convection in general circulation models (GCMs) remains a challenge. The significant biases in tropical clouds and

Corresponding author address: Jackson Tan, School of Mathematical Sciences, Monash University, Clayton, VIC 3800, Australia. E-mail: jackson.tan@monash.edu precipitation (Soden and Held 2006; Bauer et al. 2011), as well as the poor simulation of tropical variability on diurnal, intraseasonal, and interannual time scales (e.g., Yang and Slingo 2001; Neale and Slingo 2003; Lin et al. 2006), have all been linked to convective processes in models. Because of the coarse resolution of global models, convection must be represented through parameterization schemes. One of the key assumptions in most existing schemes is that convection in a grid box can be expressed diagnostically (i.e., without explicitly considering the spatial and temporal organization beyond a single grid box and the current time step). Instead, such organization is assumed to be achieved through the resolved model equations. This study aims to present a new framework for the description of the large-scale organization of convection (defined here as beyond a single GCM grid box) using satellite observations, thereby providing an avenue for testing models.

Different states of convection are associated with different cloud types as is evident from the many schematic 
depictions of convective systems [e.g., Mapes et al. (2006), and references therein]. Consequently, cloud observations provide a natural window for observing atmospheric convection. The International Satellite Cloud Climatology Project (ISCCP; Schiffer and Rossow 1983) provides a continuous global cloud climatology dating back to 1983, supplying a potentially useful long-term dataset of cloud patterns that can be exploited in studying tropical convection. Several methods of classifying ISCCP cloud fields have been developed, but they essentially fall into two categories. The first set of methods employs dynamic and/or thermodynamic parameters that are chosen to composite with cloud fields. Numerous studies have used a variety of parameters including sea level pressure (Tselioudis et al. 2000), vertical velocity (Norris and Weaver 2001; Tselioudis and Jakob 2002; Bony et al. 2004), a combination of vertical velocity and sea surface temperature (Williams et al. 2003; Ringer and Allan 2004), and tropospheric stability (Williams et al. 2006). However, this requires a subjective selection of atmospheric variables. A second approach to classifying cloud data from ISCCP, first introduced by Jakob and Tselioudis (2003), relies on the application of statistical algorithms directly to the cloud data to detect repeating patterns or regimes. Applying a clustering algorithm to joint histograms of cloud-top pressure (CTP) and optical thickness $\tau$ for $280 \mathrm{~km} \times 280 \mathrm{~km}$ grid boxes, they showed that the ISCCP cloud fields divide into several clusters or cloud regimes (also referred to as "weather states"), identifying recurring cloud patterns in the atmosphere. This technique has since been successfully applied to several geographical regions (e.g., Rossow et al. 2005; Protat et al. 2011) and has been used to investigate the association of cloud regimes with distinct meteorological conditions in the tropical western Pacific Ocean (TWP; Jakob et al. 2005; Jakob and Schumacher 2008), to evaluate the performance of climate models (Gordon et al. 2005; Williams and Tselioudis 2007; Williams and Webb 2009), and to provide further insights into atmospheric phenomena such as radiative-convective feedback (Lebsock et al. 2010), the Madden-Julian oscillation (Chen and Del Genio 2008; Tromeur and Rossow 2010), cloud radiative feedback (Oreopoulos and Rossow 2011), African easterly waves (Mekonnen and Rossow 2011), and precipitation characteristics (Lee et al. 2013; Rossow et al. 2013). Our study will use these cloud regimes.

Many of the above studies implicitly assumed an association of the tropical cloud regimes with the state of tropical convection without formally establishing this relationship. Jakob and Schumacher (2008) showed that a subset of the cloud regimes could be associated with different latent heating structures of convection, but their study was limited to the TWP. The main goal of this study is to more formally investigate the potential usefulness of the ISCCP cloud regimes for the study of tropical convection and its relationship to the largescale state of the tropical atmosphere. Particular questions we wish to address are (i) whether the ISCCP cloud regimes can provide an observational framework for convection that gives information in addition to the more commonly used outgoing longwave radiation (OLR; e.g., Waliser et al. 1993) and (ii) how the convective states identified through their cloud signatures relate to the dynamic and thermodynamic state of the tropical atmosphere. As the ISCCP cloud regimes represent cloud distributions in areas similar to a coarse-resolution GCM, the latter question is of particular relevance to their potential application in the evaluation of the representation of convection in models as well as the study of the organization of convective systems on scales larger than a model grid box.

We describe the data and analysis methods used in this study in the following section. Section 3 is devoted to identifying how the ISCCP cloud regimes relate to more conventional measures of convective activity, namely, OLR and daily precipitation, with the goal of demonstrating their utility in describing tropical convection. In section 4, we examine the large-scale atmospheric conditions in which the different convective states occur. In particular, we investigate if there are distinct features in the large-scale conditions that can be associated with each convective state-a key assumption in the parameterization of convection. The implications of our results are discussed in section 5 before concluding in section 6 .

\section{Data sources and analysis methods}

\section{a. ISCCP cloud regimes}

The ISCCP D1 dataset provides joint histograms of the frequency of occurrence of clouds with a certain combination of CTP and optical thickness (i.e., $\tau$ ) for $280 \mathrm{~km} \times 280 \mathrm{~km}$ equal area grids globally (Rossow and Schiffer 1999). These joint histograms are statistical descriptions constructed from satellite pixels with an approximate horizontal resolution of $5 \mathrm{~km}$ at nadir. In Jakob and Tselioudis (2003), the $k$-means clustering algorithm (Anderberg 1973) is applied to the joint histograms to identify repeating patterns. In Rossow et al. (2005), this method is further improved upon by a set of criteria to determine the number of clusters. Using these methods on daytime averages of the joint histograms between $35^{\circ} \mathrm{N}$ and $35^{\circ} \mathrm{S}$ gives eight centroids or cloud regimes (Fig. 1). These eight regimes are virtually 

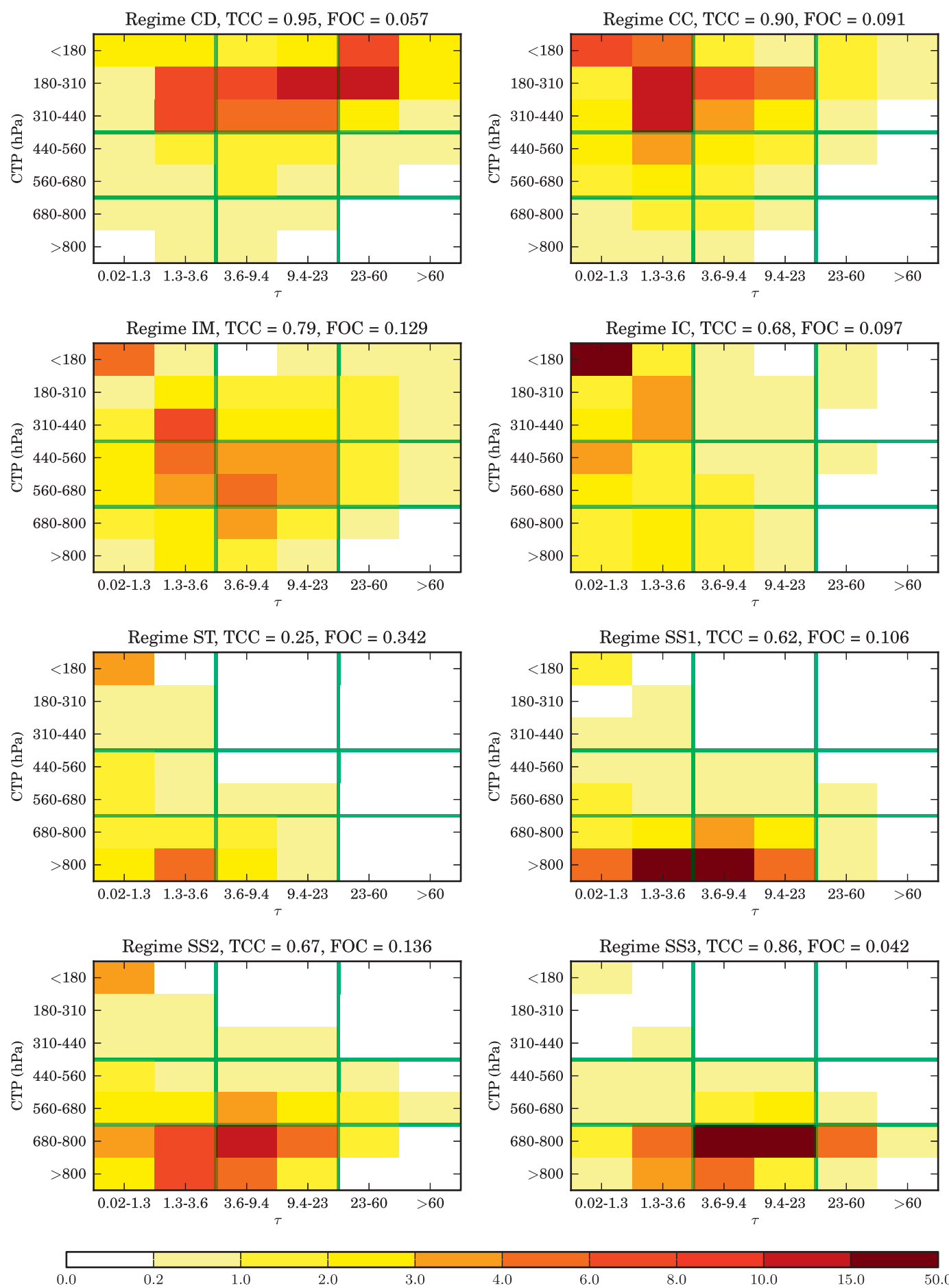

FIG. 1. ISCCP joint histograms of CTP- $\tau$ of the regime centroids. Colors indicate the cloud coverage (\%) within each CTP $-\tau$ bin. Regime acronyms are expanded in Table 1 . The TCC is obtained by summing over all bins. The relative FOC of the regime (limited to the ocean areas of our domain) is also given. The green lines approximately demarcate different cloud morphologies, with the top-right box indicating deep convective or very thick stratiform clouds (Rossow and Schiffer 1999; Hahn et al. 2001). 
TABLE 1. Names and acronyms of the eight regimes based on convective strength and dominant cloud type. The acronym follows this simple rule: the first letter denotes the convective strength and the second letter indicates the prevalent cloud type.

\begin{tabular}{|c|c|c|c|c|c|}
\hline \multicolumn{2}{|c|}{ Convective } & \multicolumn{2}{|c|}{ Intermediate } & \multicolumn{2}{|c|}{ Suppressed } \\
\hline Cloud type & Acronym & Cloud type & Acronym & Cloud type & Acronym \\
\hline Deep stratiform & $\mathrm{CD}$ & Mixture & IM & Trade cumulus & ST \\
\hline Cirrus & $\mathrm{CC}$ & Thin cirrus & IC & Stratocumulus & SS1-SS3 \\
\hline
\end{tabular}

identical to those found in Mekonnen and Rossow (2011) and Oreopoulos and Rossow (2011) (wherein they are called weather states), and many of them match those identified in earlier studies that applied a similar analysis to different areas and time periods (e.g., Jakob et al. 2005; Rossow et al. 2005; Jakob and Schumacher 2008). This agreement strongly suggests that the regimes are robust features in the cloud fields as described by the ISCCP CTP- $\tau$ histograms. To simplify our analysis, we interpolate the regime field to $2.5^{\circ} \times 2.5^{\circ}$ grids using the nearest-neighbor technique, resulting in an equal latitude-longitude grid of daily fields of cloud regimes for the entire study region.

The eight regimes describe recurring cloud patterns in the tropics and subtropics. One goal of this study is to investigate if they potentially enable the distinction between various convectively active and convectively suppressed states of the atmosphere. Based on the CTP- $\tau$ histograms of the regimes in Fig. 1, we make a first attempt at such a classification. Specifically, the presence of optically thick high-top clouds can be taken as a good indicator of deep convective or thick stratiform clouds. Such information allows us to label the cloud regimes according to their perceived convective character (convective, intermediate, or suppressed) and dominant cloud type (see Table 1). We will further investigate this assumption about the convective character of the regimes below.

The regime with the highest incidence of optically thick, high-top clouds is a convectively active regime dominated by deep stratiform cloud, labeled CD. With the highest total cloud cover (TCC) of all regimes, its joint histogram reveals signals consistent with a prevalence of towering cumulus and thick stratiform clouds that are most commonly associated with vigorous and widespread convection. However, it has a comparatively low frequency of occurrence (FOC), inhabiting primarily regions of the intertropical convergence zone and the TWP (Fig. 2). In Jakob and Schumacher (2008), this regime was found to be distinct from other regimes because of the major contribution from stratiform rain and an upward shift in the peak of the latent heating profile. The second convectively active regime shows a much lower coverage with thick clouds and a predominance of thinner, high-top cirrus clouds and is labeled CC. The presence of cirrus rather than deep stratiform clouds indicates that, while this is a convectively active regime, the convection is more isolated and likely lacks the organization that gives rise to the widespread thick clouds found in the CD regime. However, both regimes possess thick cirrus anvil, suggesting an existence of a transitional connection between them. For example, daily snapshots of the regime field (not shown) support the notion that points in the vicinity of large clusters of the $\mathrm{CD}$ regime tend to have a higher occurrence of the CC regime.

Two other regimes demonstrate convective behavior as well. However, in both of them the incidence of optically thick high-top clouds is relatively low relative to the $\mathrm{CD}$ and $\mathrm{CC}$ regimes. We classify these as intermediate regimes, indicating a likely transitional character between the deep convective regimes above and more suppressed states of the tropical atmosphere. The first intermediate regime displays a mixture of cloud types with no particular part of the histogram standing out. It is labeled as the IM regime. The second intermediate regime shows a high occurrence of very thin and high-top clouds and is labeled IC. On the one hand, this persistence of very thin cirrus may be an artifact of the "visible adjustment" in the ISCCP data processing [see section 3.3 of Marchand et al. (2010) and section 3c of Pincus et al. (2012)]. On the other hand, a comparison with ground-based active remote sensing measurements has shown that the IC regime has an elevated frequency of occurrence of thin cirrus cloud over other regimes [Jakob et al. (2005), where it is called STC]. Therefore, the nature of the IC regime should be treated with caution. Both of the intermediate regimes have a higher FOC but lower TCC than the convectively active regimes.

The remaining four regimes show no thick high-top clouds in their $\mathrm{CTP}-\tau$ histograms and are therefore classified as convectively suppressed. It is evident that one of those regimes possesses a small TCC, while the other three have relatively larger TCC. The low TCC regime is likely dominated by trade cumulus clouds and is labeled ST. It is the most frequent regime both in the tropics and subtropics, and its geographic distribution corresponds to the trade wind regions (Fig. 2). The three remaining regimes are suppressed regimes dominated 

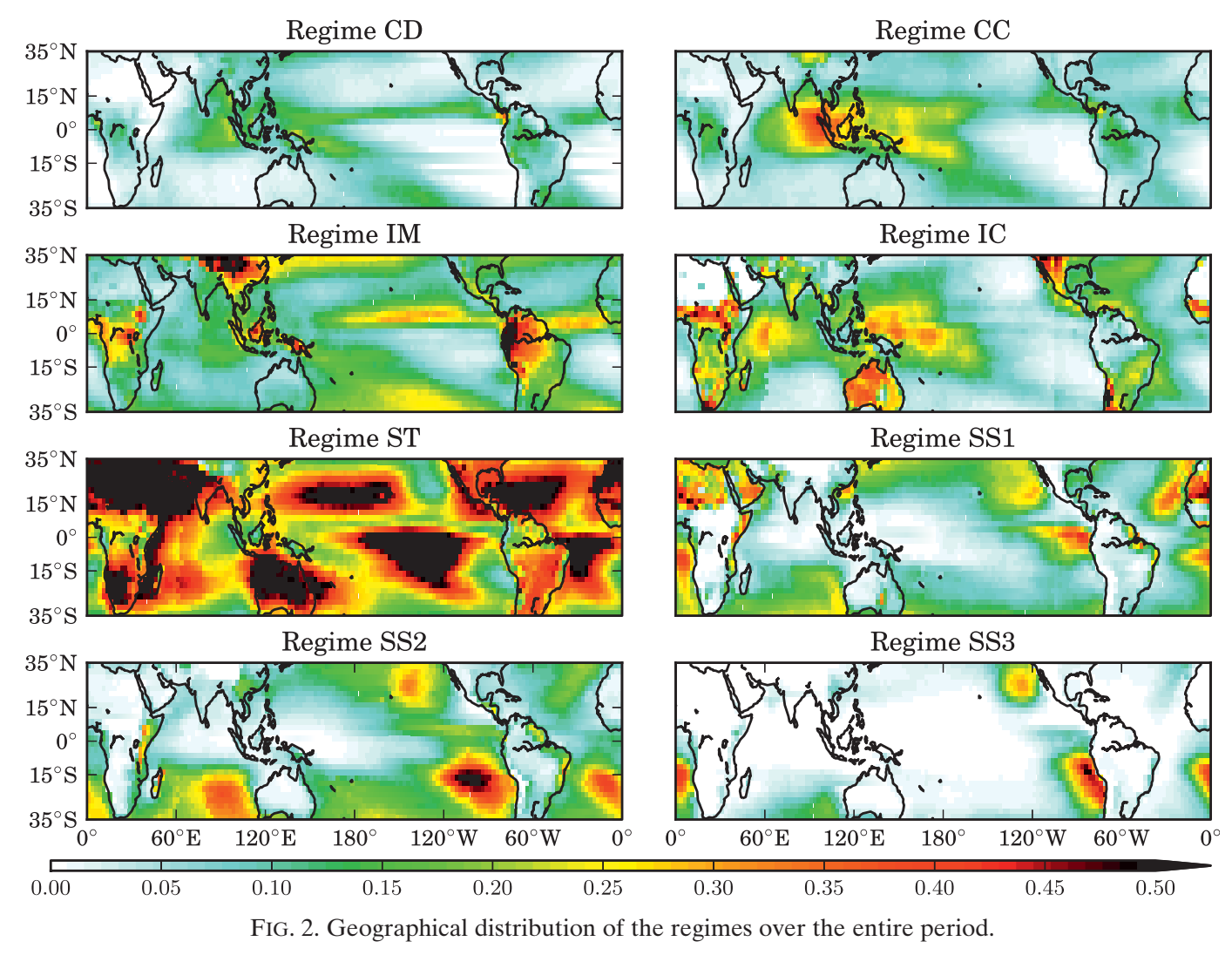

by stratocumulus clouds in various stages of their development and are labeled SS1, SS2, and SS3. These SS regimes generally occur over cold sea surface temperatures in upwelling regions to the west of the main continents (Fig. 2), which have been identified as the main stratocumulus regions on Earth in previous studies (e.g., Klein and Hartmann 1993). Although they are important features of the tropical atmosphere, our focus here is on convection, so the suppressed regimes, especially the differences among them, will not be elaborated in detail.

The satellite-based cloud regimes are derived over all surfaces. However, over land the ISCCP data suffers from orographic artifacts caused by the use of pressure as the vertical coordinate. For instance, the IM regime occurs frequently over high orography such as the islands of the Maritime Continent and the Andes ranges. Here, the clouds identified at "midlevel" are very close to the surface and are likely to be morphologically different from those in the same regime over the tropical ocean. Furthermore, the ISCCP joint histograms can only be derived during the sunlit hours of the day, so the "daily mean" joint histograms used in this study comprise daytime samples only. Over land, this daytime averaging will bias the results because of strong diurnal variations in cloudiness. To avoid misinterpretations of this kind, we restrict the analysis in this study to ocean grid boxes only (defined in ISCCP as no more than 35\% of pixels over land), where diurnal differences in cloudiness are more subdued. Work on the application of these regimes of convection over tropical land is in progress.

\section{b. Large-scale dynamic and thermodynamic quantities}

One of the main goals of our study is to investigate key relationships of the cloud regimes to the large-scale state of the tropical atmosphere. The main source for such information in this study is the European Centre for Medium-Range Weather Forecasts (ECMWF) Interim Re-Analysis (ERA-Interim; Dee et al. 2011). We use daily averages of the quantities and interpolate all variables from their original $1.5^{\circ}$ spatial resolution to a regular $2.5^{\circ}$ grid matching the ISCCP grid above. Additional tests (not included here) show that our results are insensitive to the inclusion of nighttime values in the averaging. We focus on three key aspects of the large-scale state that are associated with tropical convection: moisture, stability, and grid-mean vertical motion.

The humidity variable used is saturation fraction $r$ introduced by Bretherton et al. (2004). It is defined as the 
ratio of the vertical integral of specific humidity $q$ to that of saturated specific humidity $q_{s}$ :

$$
r=\frac{\int q d p}{\int q_{s} d p} .
$$

Bretherton et al. (2004) showed that unlike other measures of humidity such as specific humidity at a certain height or total column water vapor, $r$ is relatively independent of sea surface temperature, while exhibiting a strong relationship with precipitation that is invariant over different ocean basins. It is worth noting that the main source of variability in $r$ is midtropospheric humidity, making $r$ a potentially useful indicator of convective regime.

To describe the dynamical environment of the cloud regimes we use vertical velocity $\omega\left(\mathrm{Pa} \mathrm{s}^{-1}\right)$ at $600 \mathrm{hPa}$. A highly negative $\omega$ reveals an environment with largescale ascending motion, a condition we expect to be associated with deep convection in the atmosphere; positive $\omega$, on the other hand, is indicative of largescale subsidence.

We use two different measures of tropospheric stability in our investigation of convective regime. First, we apply the well-known lower tropospheric stability index (LTS) defined as the difference in dry potential temperature $\theta$ between 700 and $1000 \mathrm{hPa}$ :

$$
\operatorname{LTS}=\theta_{700}-\theta_{1000}
$$

Klein and Hartmann (1993) demonstrated the efficacy of this index as an indicator of low clouds, so we expect this measure to potentially relate well to the suppressed cloud regimes. However, as LTS is not a function of convective strength, it is improbable that this measure can adequately distinguish different convective regimes. As an alternative, we introduce a simple measure of deep convective instability, the modified $K$ index (Charba 1977), defined as

$$
K=\frac{T_{1000}+T_{850}}{2}-T_{500}+\frac{T_{d_{1000}}+T_{d_{850}}}{2}-T_{700}+T_{d_{700}} .
$$

where $T_{p}$ and $T_{d_{p}}$ are the temperature and dewpoint at $p(\mathrm{hPa})$, respectively. The variable $K$ is frequently used in the weather forecast community as an index for the likely presence of convective showers and thunderstorms. We choose $K$ over more common measures of stability such as convective available potential energy (CAPE) mainly for its simplicity, in part driven by our desire to apply the techniques developed in this study to global models in the future, which tend to store information only for a few levels in the atmosphere.

We use a small set of other variables to relate the ISCCP-based cloud regimes to more conventional measures of convection. Specifically, we utilize observations of the OLR at the top of atmosphere and daily precipitation $P$. The former is obtained from the ISCCP flux data (Zhang et al. 2004), while the latter uses the daily data from the Global Precipitation Climatology Project One-Degree Daily dataset (Huffman et al. 2001). All variables are interpolated onto the $2.5^{\circ} \times 2.5^{\circ}$ ISCCP grid, thus they represent values averaged over the domain of the grid.

All of our analyses use the years 1989-2007 except for $P$ where the data are only available from 1997 onward. As we intend to composite variables into the eight cloud regimes, each grid box for each day represents an individual sample. Consequently, the sample size for even the least frequent regimes is $10^{5}$ or more, ensuring that the results for all regimes are statistically robust. This is confirmed by randomly subsampling within each regime, for which we find no differences to the main conclusions presented below.

\section{Cloud regimes as convective proxies}

A key goal of this study is to investigate if the cloud regimes identified from the ISCCP dataset can be used as proxies for the state of tropical convection. We begin by using two frequently used measures of convective activity in the tropics: OLR at the top of atmosphere and $P$. A low OLR and high $P$ are usually taken as indicators of an atmosphere with strong convection, while high OLR and low $P$ indicate suppressed conditions. Figure 3 shows box-whisker diagrams for the composites of grid-averaged values of OLR and $P$ as a function of the eight regimes. It is immediately evident that the convective and intermediate regimes can be distinguished from the suppressed regimes with both lower OLR and higher $P$ values. A distinct ordering of the convective and intermediate regimes is discernible. The highest $P$ and lowest OLR are associated with the CD regime, followed by the $\mathrm{CC}$ regime. The average $P$ decreases and the average OLR increases from the convective through the intermediate regimes, first IM then IC, to the suppressed regimes. The suppressed regimes show a comparable behavior among each other, indicating that domain-averaged OLR and $P$ are ill suited in distinguishing different types of suppressed conditions. Our results are similar to Lee et al. (2013), who use different techniques and datasets in investigating the precipitation of the cloud regimes. 

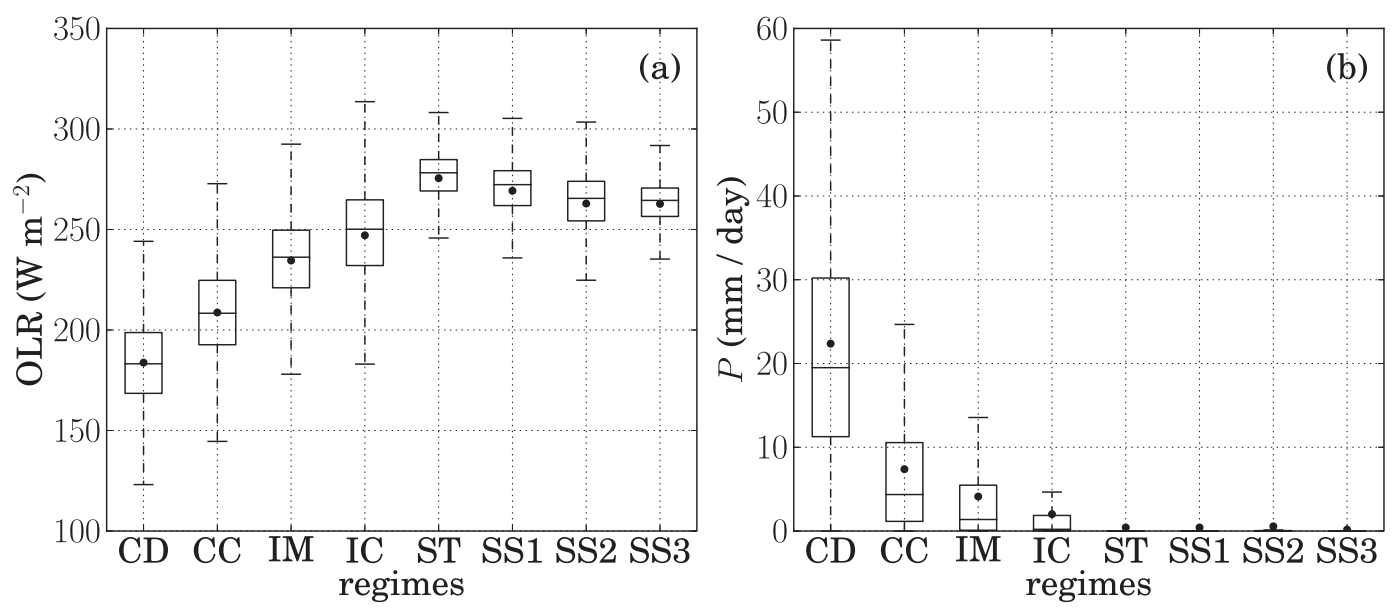

FIG. 3. Box-whisker plots with median (line), mean (point), interquartile range (box), and farthest data point within

1.5 times of the interquartile range (whiskers) of the composites with (a) OLR at the top of atmosphere or (b) $P$.

While there are significant differences between the convective and intermediate regimes in both their OLR and $P$ distributions, there is also some degrees of overlap. To further elucidate how well the regimes distinguish in these measures of convective strength, we investigate their locations in OLR- $P$ space. Figure 4 shows $2 \mathrm{D}$ histograms for the frequency of occurrences of the convective and intermediate regimes in the OLR $-P$ parameter space (suppressed regimes are neglected because they generally have no precipitation). The most striking feature of Fig. 4 is the substantially distinct pattern the $\mathrm{CD}$ regime has over all other regimes. Its most common rainfall bins are above $10 \mathrm{~mm}_{\text {day }}{ }^{-1}$. The other three regimes have much lower rainfall, with the most frequent bin in the lowest $P$ row, and show a progression to higher OLR as expected in Fig. 3a. Figure 4 enables us to distinguish the convective qualities between the regimes. For example, while the $\mathrm{CD}$ and $\mathrm{CC}$ regimes have overlapping OLR values, they clearly deviate from each other in $P$. In contrast, the IM and IC regimes have similar $P$ profiles, but their OLR distributions have a stronger contrast. This highlights the feasibility of the ISCCPbased cloud regimes as alternative convective proxies, as they are physically based objective tools to delineate between different convective environments that may have similar grid-averaged value of OLR or $P$.

Figures $3 \mathrm{~b}$ and 4 have already illustrated the very different precipitation behaviors of the cloud regimes. Further insight into this behavior is provided by Fig. 5, which shows the fraction of days in each regime for which rainfall exceeds the thresholds of $0.1,1$, and $10 \mathrm{~mm} \mathrm{day}^{-1}$. These values represent spatial averages for grid boxes of $2.5^{\circ} \times 2.5^{\circ}$; as such they were chosen to indicate any, light, and heavy precipitation, respectively. The figure also shows the overall contribution of each regime to the total tropical rainfall. As we would expect, the $\mathrm{CD}$ regime shows the most frequent occurrences of precipitation, with nearly all of them associated with at least light rainfall and, remarkably, close to $80 \%$ with heavy rainfall. As mentioned previously, $\mathrm{CD}$ is the only regime in which stratiform precipitation composes more than $50 \%$ of the total rainfall in the TWP (Jakob and Schumacher 2008). Notably, the CD regime contributes $45 \%$ to tropical precipitation even though it occurs only $5.7 \%$ of the time. The CC regime shows a higher than $90 \%$ frequency of occurrence of rainfall, justifying its identification as a convective regime. However, the rainfall is heavy only $25 \%$ of the time. The intermediate regimes show rainfall frequencies between $55 \%$ and $75 \%$, but most of these events are light. The suppressed regimes ST and SS, which from their cloud signature are primarily associated with trade cumulus and stratocumulus, respectively, have occasionally light rainfall and rarely heavy precipitation.

We have combined a regime classification of tropical cloudiness with traditional measures of the character of tropical convection. The results confirm our assertion that cloud regimes identified from ISCCP do exhibit a spectrum of convective strengths and can be used to describe the states of tropical convection. Moreover, we have shown that using the cloud regimes provides additional information over the use of OLR alone, and enables the distinction between different suppressed states. In particular, we conjecture that three of the cloud regimes signify the three "stretched building blocks" of tropical convection summarized by Mapes et al. (2006), a notion which will be elaborated on further in section 5. These attributes of the regimes allow them to be used in investigations of the large-scale behavior of tropical convection, as an alternative to more 

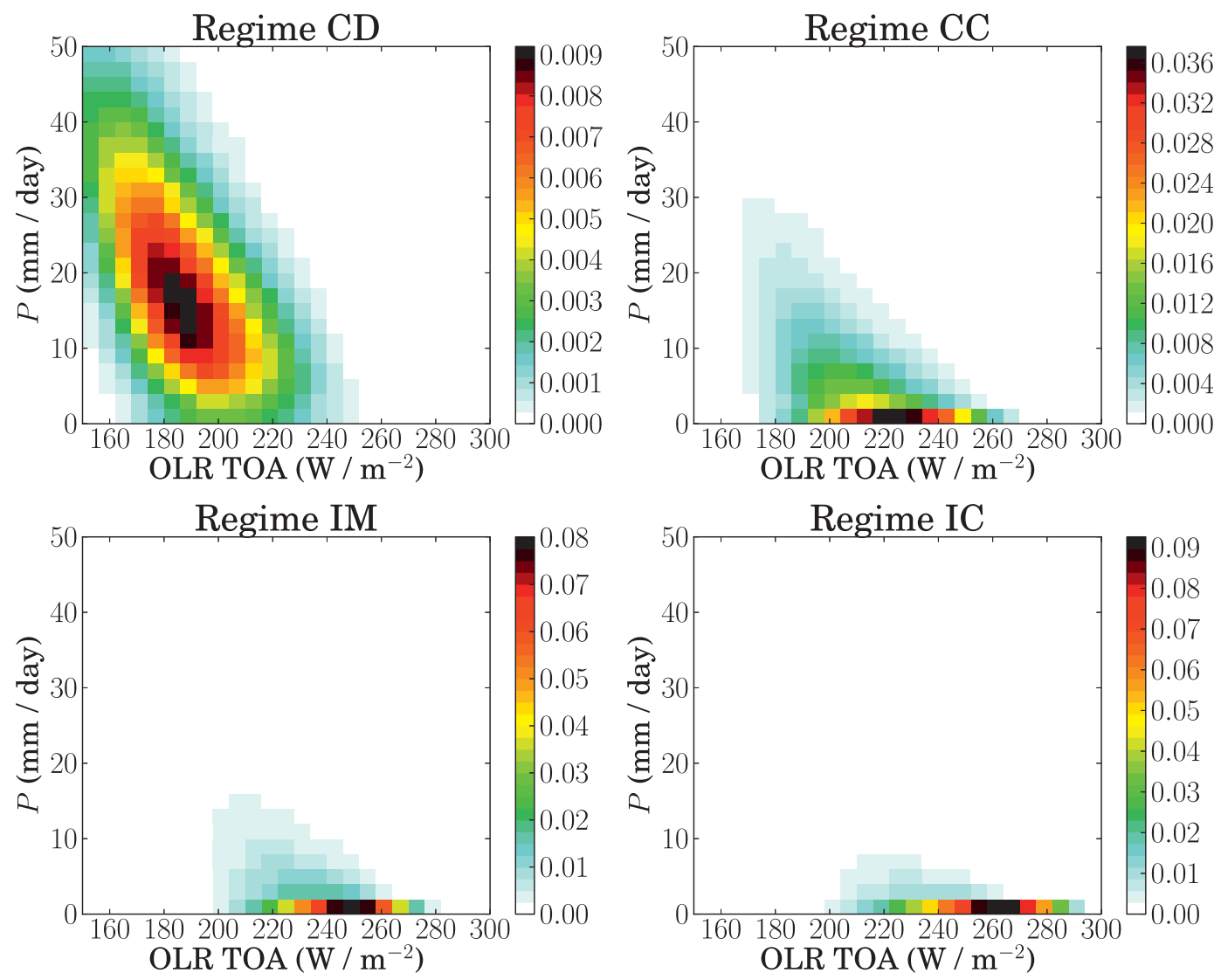

FIG. 4. 2D plots of the FOC of the CD, CC, IM, and IC regimes in the OLR- $P$ parameter space. All the bins within each $2 \mathrm{D}$ histogram sum to one. Note that the lowest $P$ bin contains $P=0$. Suppressed regimes are omitted because they are generally nonprecipitating.

traditionally used measures such as outgoing longwave radiation at the top of atmosphere or precipitation.

\section{The large-scale environment of convection}

Having established that the states of tropical convection can be described by ISCCP-based cloud regimes, we now investigate their relationship to key large-scale atmospheric variables. Figure 6 shows box-whisker representations of the distributions of saturation fraction (i.e., $r$ ), vertical velocity at $600 \mathrm{hPa}$ (i.e., $\omega$ ), modified $K$ index (i.e., $K$ ), and LTS within each regime. These composites are derived from all months, but the relationships remain robust over different seasons. As a statistical measure of the distinction between the distributions, we apply the Kolmogorov-Smirnov twosample (KS2) test to composites from different pairs of regimes. The KS2 test is a nonparametric and distribution-free statistical approach for comparing if two samples are drawn from the same population. To avoid spatial and temporal autocorrelation in the data, all our KS2 tests randomly sample 2000 composite values (out of approximately 90000 ) from each month to a total of 456000 values.

Figure 6a exhibits a strong distinction between the convective/intermediate and the suppressed regimes in

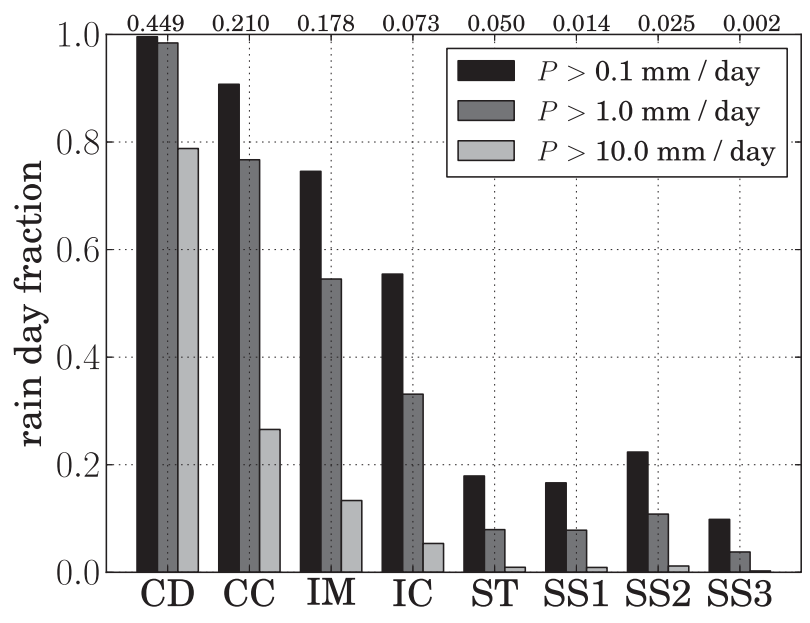

FIG. 5. Fraction of days with rain above certain thresholds. Values at the top denote the fractional contribution of the regime to overall precipitation. 

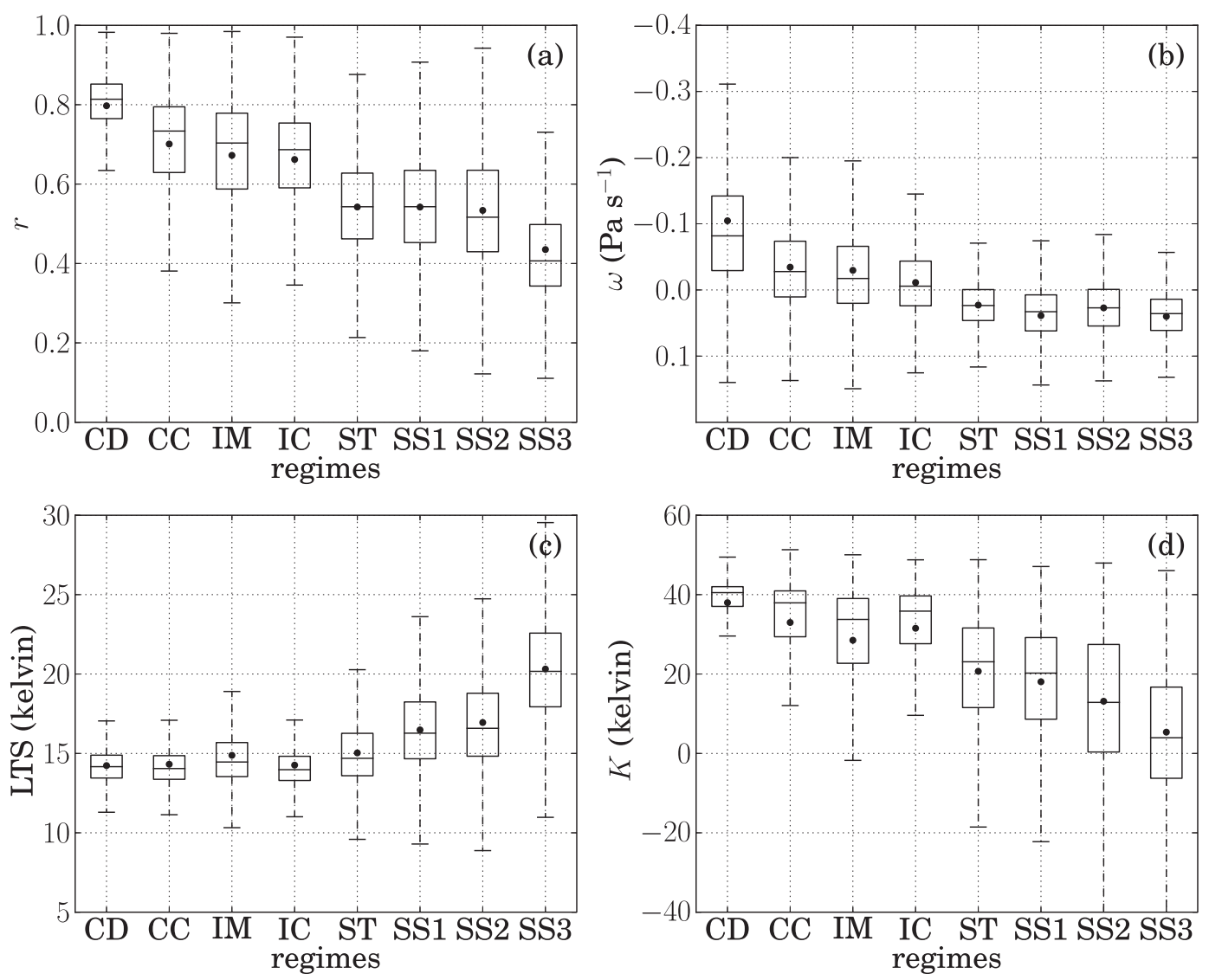

FIG. 6. Box-whisker plots with median (line), mean (point), interquartile range (box), and farthest data point within 1.5 times of the interquartile range (whiskers) of the composites with (a) $r$, (b) $\omega$, (c) LTS, and (d) $K$.

their $r$ distributions. Once again, the CD stands out as a very wet regime, with a median above $r=0.8$ and comparatively small variability around this value. The $\mathrm{CC}$ and the intermediate regimes IM and IC show distributions of $r$ that are fairly similar to each other with a slight decrease in median from CC to IC. Likewise most of the suppressed regimes share similar $r$ distributions with the exception of the SS3 regime, which is the driest of the eight regimes. Despite some apparent similarity in Fig. 6a, the KS2 tests reject the null hypothesis that any of the two $r$ distributions are drawn from the same distribution ( $p$ value $<0.01$ ).

The $\omega$ distributions (Fig. 6b) also show a good distinction between the convection and the suppressed regimes, with the median values of both the convective and intermediate regimes showing ascending values (negative $\omega$ ), while the median values for the suppressed regimes indicate subsiding conditions. Furthermore, more than $75 \%$ of the distribution in each suppressed regime resides in environments with descending motion. The opposite is not true for the intermediate regimes. The distribution for the IC regime shows a nearly equal division between upward and downward motion and the IM regime shows a slight preference for existing under upward-moving large-scale conditions. The CC regime's $\omega$ distribution is very similar to that of the IM regime. It is the $\mathrm{CD}$ regime that shows a very clear preference for upward motion not only with the most negative median, but also the largest "extreme" values. This is entirely consistent with the high precipitation in this regime as highlighted in section 3 . As with $r, \mathrm{KS} 2$ tests conclude that the distributions are all distinct ( $p$ value $<0.01$ ). It is worth noting that of the largescale variables investigated, all of which are derived from the ERA-Interim, $\omega$ carries by far the largest uncertainty as the analysis of the associated divergent wind field in numerical weather prediction systems remains challenging.

The composites of the stability indices (Figs. 6c and 6d) show a good distinction of the convective/intermediate regimes from the suppressed regimes. Within each of the two groups, the distinction by stability is stronger for the suppressed regimes. In particular, using LTS it is possible to distinguish the ST regime as the suppressed 
regime with the weakest LTS as well as the SS3 regime as clearly exhibiting the largest values. The CD, CC, and IC regimes, on the other hand, are seemingly identical in LTS, an observation that may reflect their lack of low clouds (Fig. 1). However, the IM regime has a comparable occurrence of low clouds, yet it resides in an environment that has visibly higher LTS. This implies the failure of LTS to distinguish between different states of convection, much like how $r$ or $\omega$ is for suppressed regimes. There is a slight improvement in using $K$ over LTS for convective/intermediate regimes, but this comes at the expense of a lower distinction between suppressed regimes. This implies that convection-or at least its cloud structures - is more closely related to vertical motion (or convergence) and humidity than stability. This is particularly true for the very strong convection represented by the CD regime. Again, no two distributions are from the same underlying population according to KS2 tests with a $p$ value less than 0.01 , even for the apparently similar CD, CC, and IC regimes in LTS.

It is evident from Fig. 6 that all composites exhibit a considerable spread. Even for the CD regime, which shows the largest distinction of the convective regimes, there is a nonnegligible number of values that fall into the low $r$ and positive $\omega$ range. While some of this variability might be explained by the fact that the atmospheric variables are drawn from an imperfect numerical analysis system, it is plausible that the variability is an inherent feature of tropical convection at this scale. The, at least partially, stochastic nature of the relationship of tropical convection to large-scale conditions has been the subject of much discussion in the recent literature (e.g., Lin and Neelin 2000; Neelin et al. 2008; Plant and Craig 2008; Stechmann and Neelin 2011; Jakob et al. 2011; Frenkel et al. 2012). Our results are consistent with the notion that while there is clearly distinguishable influence of the large-scale state on convection, the relationship is far from deterministic and includes considerable element of stochastic behavior.

In section 3 , the advantage of assessing multivariate relationships was evident by the use of Fig. 4. Here, we explore this possibility by combining three of the four large-scale variables into a single diagram, as exemplified by Fig. 7. Here, the $K$ and $\omega$ values of each data point are indicated by its location in the diagram and its $r$ value by the color of the dot. The interquartile range is indicated by the crosses in the diagram for $K$ and $\omega$ and on the color bar for $r$. Figure 7 uses all data points independent of regime, exhibiting well-defined relationships between the variables. For example, data points with strong ascending motion tend to be wet, while highly stable points tend to be very dry, a reflection of the differences in the character of the ascending and descending

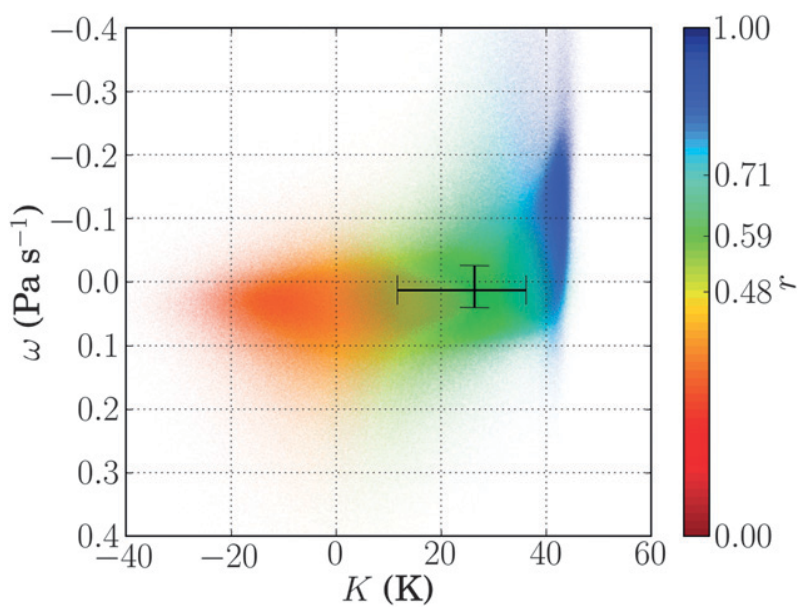

FIG. 7. A scatter diagram of the relationship between $K$ ( $x$ axis), $\omega$ ( $y$ axis), and $r$ (color) for all regimes. The quartiles are marked by the crosses and on the color bar.

branches of the atmospheric circulation in the tropics and subtropics.

Figure 8 shows the scatter diagrams for each regime. As expected from the earlier results, the CD regime populates the least stable, most strongly ascending, and most humid part of the parameter space spanned by $K, \omega$, and $r$. The CC regime begins to include points of more positive vertical velocities and weaker instability as well as drier atmospheric states, a trend that continues for the IM regime. The suppressed regimes, and to some extent the IC regime as well, show a distinctly different shape in the distribution of points, which shifts to an increasingly horizontal alignment when transitioning through the ST regime to the SS regimes. As one may expect, most of the points in the suppressed regimes populate the descending and stable part of the diagram along with much lower values of $r$. The transition from a mostly vertical alignment of points in the CD regime to a mostly horizontal alignment for the suppressed regimes indicates that the three large-scale variables displayed taken together show some skill in distinguishing between the convective states of the tropical atmosphere as represented by the cloud regimes. It is worth remembering that no large-scale information was utilized in the definition of the regimes, which are based on cloud information alone.

When comparing Fig. 8 with Fig. 6, we can also identify features of the regimes that are not easily visible when the composites are calculated for each variable separately. For example, all regimes have a significant number of points in the $\omega=\sim 0 \mathrm{~Pa} \mathrm{~s}^{-1}$ environment, but those in the CD regime are generally moist while suppressed regimes show a range of $r$ associated with the same condition. Also, points with negative $\omega$ tend to 

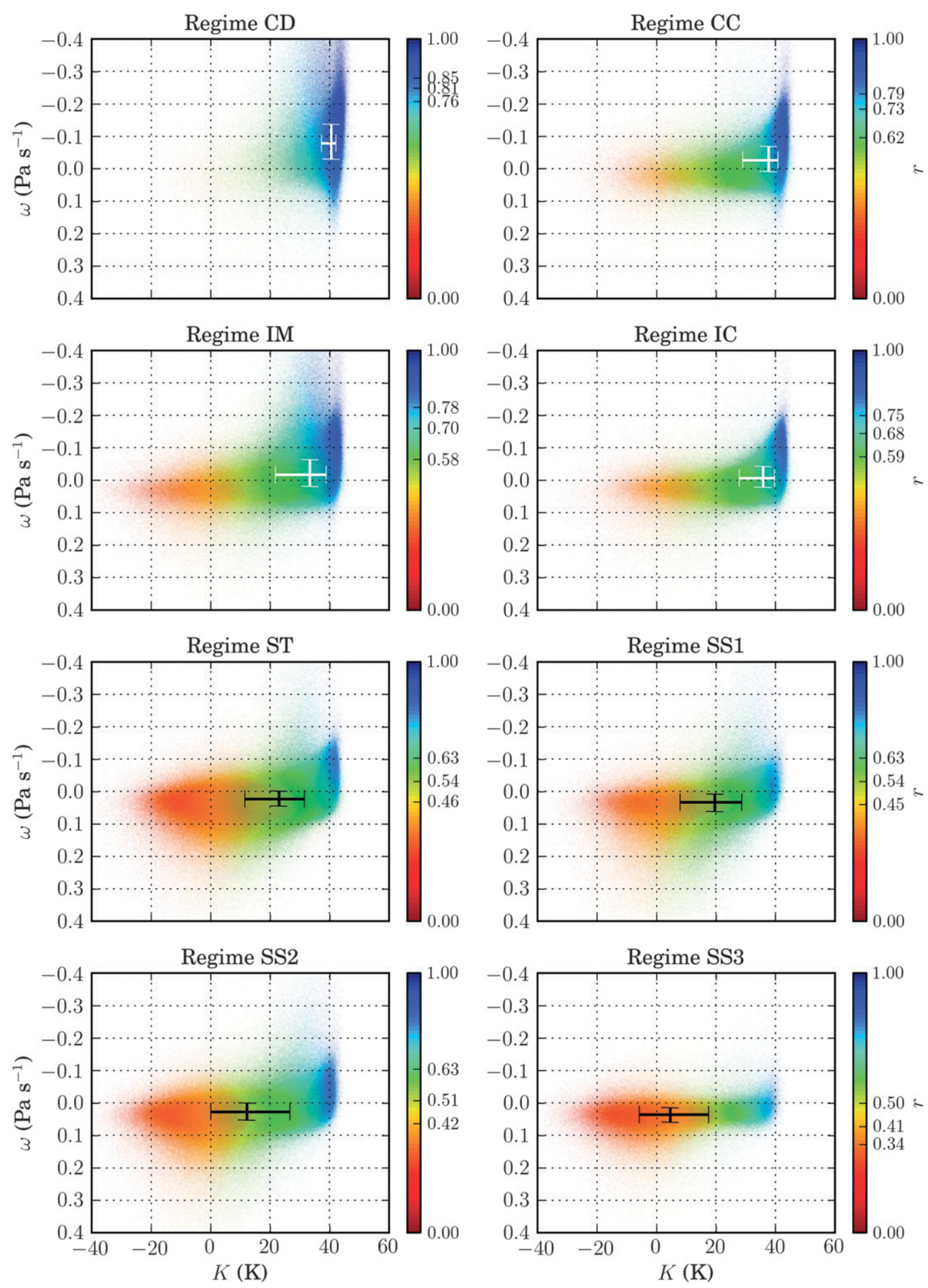

FIG. 8. As in Fig. 7, but for each regime.

occur with high $K$ and $r$ in the convective regimes, but with lower $K$ and $r$ in the suppressed regimes.

Combining the results of the previous two sections demonstrates that using the objectively identified
ISCCP-based cloud regimes as convective proxies leads to a reasonable distinction of large-scale states for each of the regimes. This highlights the presence of a deterministic element in the large-scale to convection 

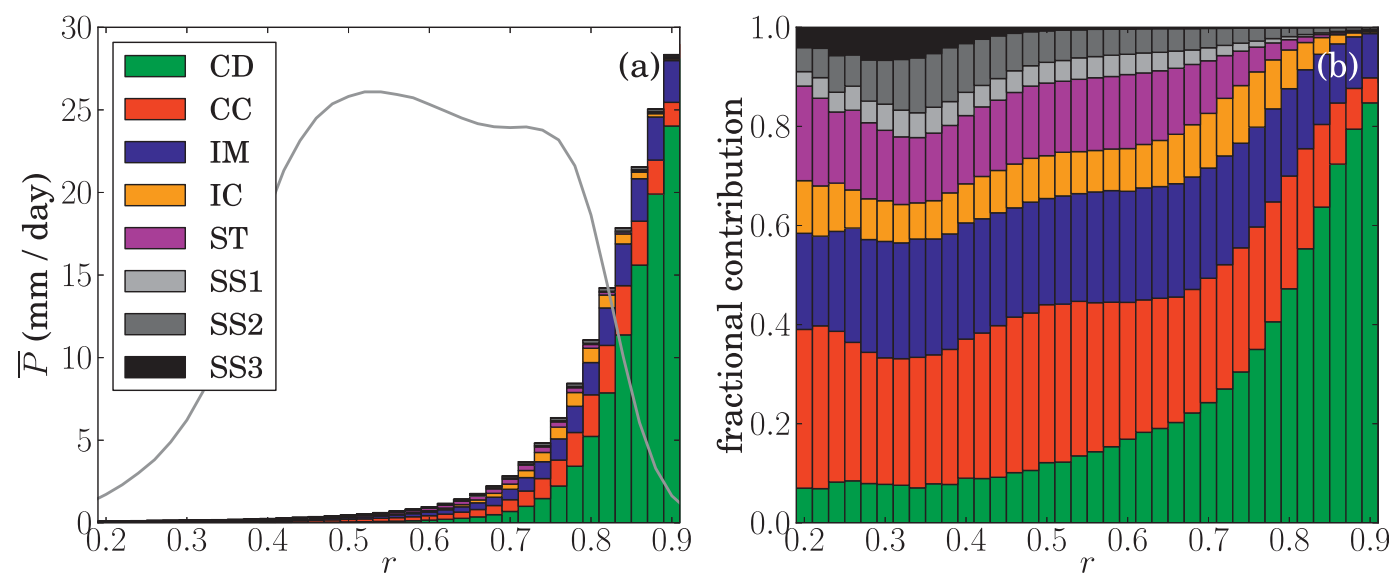

FIG. 9. (a) A histogram of the mean daily precipitation $\bar{P}$ in the tropics and subtropics for each bin of $r$ by each regime. The gray line indicates the relative number of data points in each bin of $r$. (b) The fractional contribution of each regime to each bin of $r$.

relationship. At the same time our results show that there is a substantial amount of scatter in those relationships, a possible sign of the considerable influence of stochastic processes on the convective state of the tropical atmosphere given a specific configuration of the large-scale state.

\section{Discussion}

In sections 3 and 4, we examined the convective strengths and large-scale atmospheric conditions of eight ISCCP-based cloud regimes through composite analysis. The regimes, obtained by applying an a priori clustering algorithm to joint histograms of cloud-top pressure and optical thickness, describe recurring cloud patterns in the tropics and subtropics. We have shown that the regimes can be interpreted as empirical archetypes of the convective state of the tropical atmosphere, each with distinct large-scale environmental conditions.

Our results quantify the $\mathrm{CD}$ regime as a wet, thermodynamically unstable and heavily precipitating regime with large-scale ascending motions. This is consistent with the signature of extensive thick stratiform clouds in its joint histogram (Fig. 1). With a high frequency of occurrence in the intertropical convergence zone and tropical western Pacific and a $45 \%$ contribution to tropical precipitation, this regime is likely associated with more organized forms of convection including a large stratiform component (Jakob and Schumacher 2008). The CC is a regime of less organized deep convection, as it displays weaker precipitation, a drier environment, and a prevalence of cirrus rather than thick stratiform clouds. The IM regime shows a mixture of coexisting cloud types including some coverage with midlevel-top congestus and altocumulus or altostratus clouds.
From these results, we conjecture that the $\mathrm{CD}, \mathrm{CC}$, and IM regimes are observationally based representations of the three stretched building blocks of precipitating tropical convection postulated by Mapes et al. (2006). Here, the CD regime represents strong convection with large stratiform clouds and heavy rainfall, the "stratiform" mode of a tropical cloud mixture. The $\mathrm{CC}$ regime on the other hand is more typical of deep convection without significant stratiform componentsthe "convective" mode. The IM regime is a transitional regime from suppressed to active conditions with a frequent occurrence of cloud tops at midlevels-the "congestus" mode of tropical convection.

To provide an example for the potential new insight that can be gleaned from using the cloud regimes as convective proxies, we use them to investigate the strong nonlinear relationship between saturation fraction and precipitation over the tropical oceans. Bretherton et al. (2004) and Holloway and Neelin (2009) have shown that there is little rainfall in the tropics when $r$ is low. A distinct increase in precipitation occurs at values of $r$ from around 0.5 to 0.6 and a sharp increase of rainfall at values above 0.7 . As much of the rainfall in the tropics is associated with convective systems, this behavior is usually interpreted as a strong relationship of convection with the relative humidity of atmosphere, and in particular the midtroposphere (Holloway and Neelin 2009).

An interesting feature of the distinction of the largescale atmospheric states by cloud regime carried out above was the strong distinction of the convective regimes, in particular the $\mathrm{CD}$ regime, in both rainfall (Figs. 3b and 4) and $r$ (Figs. 6a and 8). Figure 9a shows how each of the cloud regimes contributes to the wellestablished relationship between rainfall and $r$. The 
sharp increase of rainfall at $r \approx 0.7$ is evident. Figure $9 \mathrm{~b}$ shows the relative contribution of each regime to this behavior. At low $r$, the main contributors to the (very low) values of rainfall are the CC, IM, and ST regimes. This behavior remains roughly constant with increasing $r$ until the rapid rise in precipitation at $r \approx 0.7$. It is evident that this increase is caused by a corresponding increase in the contribution to rainfall by the $\mathrm{CD}$ regime, which is $20 \%$ or less at $r<0.7$ and increases sharply to about $80 \%$ at $r=0.9$. In short, it is strong, organized convection with large areas of stratiform clouds that almost entirely drives the strong increase of rainfall in moist tropical atmospheres, an important insight gained by the use the cloud regimes as convective proxies.

The reasonably strong relationship of the regimes to the large-scale state of the atmosphere gives rise to other potential applications such as GCM evaluation as well as the use of these relationships to statistically model the large-scale distribution of the key states of tropical convection. The latter would enable the testing of some of the key assumptions in the treatment of convection in weather and climate models. Among them is the premise of the entirely diagnostic relationships between convective and large-scale states (i.e., the convective state in each grid box is usually independent of convection in the previous time step or neighboring grid boxes). A statistical model based on the cloud regimes and their relationships to the larger scales would allow us to assess the validity of these assumptions as observed spatial and temporal coherence can be introduced or omitted from the model to investigate their importance. One could even envisage introducing such a model into GCMs to complement the existing treatment of cumulus convection, similar to what was done on small scales in the study of Bengtsson et al. (2011) but on scales above a model grid box.

\section{Summary and conclusions}

The main aim of this study was to investigate if regimes of tropical cloudiness objectively derived from ISCCP data on scales of $280 \mathrm{~km} \times 280 \mathrm{~km}$ can be applied as proxies for tropical convection. Most importantly, it was our goal to establish if doing so provides additional information on convection and its relationship to the large-scale state of the atmosphere than when using more commonly applied measures of convective strength, such as outgoing longwave radiation and precipitation.

Regime composite analyses of grid-averaged values of OLR and $P$, as well as the large-scale parameters of vertical motion at $600 \mathrm{hPa}$, saturation fraction, and two stability indices substantiates the grouping of the cloud regimes into three main classes: two convective regimes, four suppressed regimes, and two intermediate regimes of transitional character. As compared to conventional measures such as OLR, these regimes give additional information in their cloud structure, and are able to segregate the suppressed environments. We further showed that the two convective regimes possess very distinct characteristics from each other in terms of $P$ as well as moisture and convective instability of the environment, despite having overlapping OLR values usually associated with tropical deep convection. Together with the transitional IM regime which is characterized by a wideranging mixture of clouds with a substantial midlevel-top cloudiness, we suggest in section 5 that these three regimes provide the observational proxies of the "stretched building blocks" of tropical convection identified by Mapes et al. (2006), namely, a regime dominated by congestus convection (IM), a regime dominated of deep convection (CC), and a regime dominated by a large stratiform cloud component (CD).

The composite analysis of the large-scale state with cloud regime reveals a distinct deterministic component in the large- to small-scale relationship, in particular when a multidimensional analysis of this relationship is performed. We find that the convective regimes populate a distinctly different part of a parameter space spanned by three large-scale variables related to stability (modified $K$ index), relative humidity (saturation fraction), and vertical motion or convergence, than suppressed regimes. On the distinction of regimes within the same class, saturation fraction and vertical motion are better able to distinguish the regimes within the convective and intermediate class, while the stability indices perform better in this respect for suppressed regimes. Despite the existence of a deterministic component, there is scatter and overlap between regimes in the large-scale parameter space, suggesting a substantial stochastic component in the large- to small-scale relationships. Care must be taken in interpreting this observation, as the degree of stochastic behavior can be a strong function of the large-scale variables chosen in identifying the relationship (Jakob et al. 2011). We aimed to minimize this influence by choosing several largescale variables and performing multivariate analyses.

Finally, using the relationship between precipitation and saturation fraction we showed that the information on convective characteristics contained in the cloud regimes can be used to provide additional insight into the role of different convective regimes in this relationship. We demonstrated that the strong increase in rainfall at high saturation fraction is brought about by an increase in the occurrence of the more organized and stratiform CD regime. 
We discussed several other potential applications of the cloud regimes as proxies for convection, which include evaluating GCMs in their connections between gridbox convection and the large-scale atmosphere as well as the design of simple statistical models of tropical convection. The latter could be used to investigate the behavior of convection on very large scales as well as to inform the design of parameterization of convection in weather and climate models. Such a statistical model, making use of the properties of tropical convection highlighted in this study, is the subject of future work.

Acknowledgments. This project is funded under the Australian Research Council Centre of Excellence for Climate System Science (CE110001028). JT and CJ gratefully acknowledge the support of the Monash e-Research Centre in this work. TPL is supported by the Australian Research Council's Future Fellowship program (FT0990892). We thank the anonymous reviewers for their helpful comments and suggestions.

\section{REFERENCES}

Anderberg, M. R., 1973: Cluster Analysis for Applications. Academic Press, $359 \mathrm{pp}$

Bauer, P., G. Ohring, C. Kummerow, and T. Auligne, 2011: Assimilating satellite observations of clouds and precipitation into NWP models. Bull. Amer. Meteor. Soc., 92, ES25-ES28.

Bengtsson, L., H. Körnich, E. Källén, and G. Svensson, 2011: Large-scale dynamical response to subgrid-scale organization provided by cellular automata. J. Atmos. Sci., 68, 31323144.

Bony, S., J.-L. Dufresne, H. Le Treut, J.-J. Morcrette, and C. Senior, 2004: On dynamic and thermodynamic components of cloud changes. Climate Dyn., 22 (2-3), 71-86, doi:10.1007/ s00382-003-0369-6.

Bretherton, C. S., M. E. Peters, and L. E. Back, 2004: Relationships between water vapor path and precipitation over the tropical oceans. J. Climate, 17, 1517-1528.

Charba, J. P., 1977: Operational system for predicting thunderstorms two to six hours in advance. NOAA Tech. Memo. NWS TDL-64, $24 \mathrm{pp}$.

Chen, Y., and A. D. Del Genio, 2008: Evaluation of tropical cloud regimes in observations and a general circulation model. Climate Dyn., 32 (2-3), 355-369, doi:10.1007/s00382-008-0386-6.

Dee, D. P., and Coauthors, 2011: The ERA-Interim reanalysis: Configuration and performance of the data assimilation system. Quart. J. Roy. Meteor. Soc., 137 (656), 553-597, doi:10.1002/ qj.828.

Frenkel, Y., A. J. Majda, and B. Khouider, 2012: Using the stochastic multicloud model to improve tropical convective parameterization: A paradigm example. J. Atmos. Sci., 69, 1080-1105.

Gordon, N. D., J. R. Norris, C. P. Weaver, and S. A. Klein, 2005: Cluster analysis of cloud regimes and characteristic dynamics of midlatitude synoptic systems in observations and a model. J. Geophys. Res., 110, D15S17, doi:10.1029/2004JD005027.
Hahn, C. J., W. B. Rossow, and S. G. Warren, 2001: ISCCP cloud properties associated with standard cloud types identified in individual surface observations. J. Climate, 14, 11-28.

Holloway, C. E., and J. D. Neelin, 2009: Moisture vertical structure, column water vapor, and tropical deep convection. J. Atmos. Sci., 66, 1665-1683.

Huffman, G. J., R. F. Adler, M. M. Morrissey, D. T. Bovlin, S. Curtis, R. Joyce, B. McGavock, and J. Susskind, 2001: Global precipitation at one-degree daily resolution from multisatellite observations. J. Hydrometeor., 2, 36-50.

Jakob, C., and G. Tselioudis, 2003: Objective identification of cloud regimes in the Tropical Western Pacific. Geophys. Res. Lett., 30, 2082, doi:10.1029/2003GL018367.

, and C. Schumacher, 2008: Precipitation and latent heating characteristics of the major tropical western Pacific. J. Climate, 21, 4348-4364.

, G. Tselioudis, and T. Hume, 2005: The radiative, cloud, and thermodynamic properties of the major tropical western $\mathrm{Pa}$ cific cloud regimes. J. Climate, 18, 1203-1215.

, L. Davis, V. Kumar, and P. May, 2011: Representing convection in models-How stochastic does it need to be? Proc. Representing Model Uncertainty and Error in Weather and Climate Prediction, Reading, United Kingdom, European Centre for Medium-Range Weather Forecasts, 41-52.

Klein, S. A., and D. L. Hartmann, 1993: The seasonal cycle of low stratiform clouds. J. Climate, 6, 1587.

Lebsock, M. D., C. Kummerow, and G. L. Stephens, 2010: An observed tropical oceanic radiative-convective cloud feedback. J. Climate, 23, 2065-2078.

Lee, D., L. Oreopoulos, G. J. Huffman, W. B. Rossow, and I.-S. Kang, 2013: The precipitation characteristics of ISCCP tropical weather states. J. Climate, 26, 772-788.

Lin, J.-L., and Coauthors, 2006: Tropical intraseasonal variability in 14 IPCC AR4 climate models. Part I: Convective signals. J. Climate, 19, 2665-2690.

Lin, J. W.-B., and J. D. Neelin, 2000: Influence of a stochastic moist convective parameterization on tropical climate variability. Geophys. Res. Lett., 27 (22), 3691-3694.

Mapes, B., S. Tulich, J. Lin, and P. Zuidema, 2006: The mesoscale convection life cycle: Building block or prototype for large-scale tropical waves? Dyn. Atmos. Oceans, 42 (1-4), 3-29, doi:10.1016/j.dynatmoce.2006.03.003.

Marchand, R., T. Ackerman, M. Smyth, and W. B. Rossow, 2010: A review of cloud top height and optical depth histograms from MISR, ISCCP, and MODIS. J. Geophys. Res., 115, D16206, doi:10.1029/2009JD013422.

Mekonnen, A., and W. B. Rossow, 2011: The interaction between deep convection and easterly waves over tropical North Africa: A weather state perspective. J. Climate, 24, 4276-4294.

Neale, R., and J. Slingo, 2003: The maritime continent and its role in the global climate: A GCM study. J. Climate, 16, 834848.

Neelin, J. D., O. Peters, J. W.-B. Lin, K. Hales, and C. E. Holloway, 2008: Rethinking convective quasi-equilibrium: Observational constraints for stochastic convective schemes in climate models. Philos. Trans. Roy. Soc. London, 366A, 2581-2604, doi:10.1098/ rsta.2008.0056.

Norris, J. R., and C. P. Weaver, 2001: Improved techniques for evaluating GCM cloudiness applied to the NCAR CCM3. J. Climate, 14, 2540-2550.

Oreopoulos, L., and W. B. Rossow, 2011: The cloud radiative effects of International Satellite Cloud Climatology Project weather states. J. Geophys. Res., 116, D12202, doi:10.1029/2010JD015472. 
Pincus, R., S. Platnick, S. A. Ackerman, R. S. Hemler, and R. J. Patrick Hofmann, 2012: Reconciling simulated and observed views of clouds: MODIS, ISCCP, and the limits of instrument simulators. J. Climate, 25, 4699-4720.

Plant, R. S., and G. C. Craig, 2008: A stochastic parameterization for deep convection based on equilibrium statistics. J. Atmos. Sci., 65, 87-105.

Protat, A., J. Delanoë, P. T. May, J. Haynes, C. Jakob, E. O’Connor, M. Pope, and M. C. Wheeler, 2011: The variability of tropical ice cloud properties as a function of the large-scale context from ground-based radar-lidar observations over Darwin, Australia. Atmos. Chem. Phys., 11 (16), 8363-8384, doi:10.5194/ acp-11-8363-2011.

Ringer, M. A., and R. P. Allan, 2004: Evaluating climate model simulations of tropical cloud. Tellus, 56A, 308-327, doi:10.1111/ j.1600-0870.2004.00061.x.

Rossow, W. B., and R. A. Schiffer, 1999: Advances in understanding clouds from ISCCP. Bull. Amer. Meteor. Soc., 80, 2261-2287.

— , G. Tselioudis, A. Polak, and C. Jakob, 2005: Tropical climate described as a distribution of weather states indicated by distinct mesoscale cloud property mixtures. Geophys. Res. Lett., 32, L21812, doi:10.1029/2005GL024584.

—, A. Mekonnen, C. Pearl, and W. Goncalves, 2013: Tropical precipitation extremes. J. Climate, 26, 1457-1466.

Schiffer, R. A., and W. B. Rossow, 1983: The International Satellite Cloud Climatology Project (ISCCP)-The first project of the World Climate Research Programme. Bull. Amer. Meteor. Soc., 64, 779-784.

Soden, B. J., and I. M. Held, 2006: An assessment of climate feedbacks in coupled ocean-atmosphere models. J. Climate, 19, 3354-3360.

Stechmann, S. N., and J. D. Neelin, 2011: A stochastic model for the transition to strong convection. J. Atmos. Sci., 68, 29552970.
Tromeur, E., and W. B. Rossow, 2010: Interaction of tropical deep convection with the large-scale circulation in the MJO. J. Climate, 23, 1837-1853.

Tselioudis, G., and C. Jakob, 2002: Evaluation of midlatitude cloud properties in a weather and a climate model: Dependence on dynamic regime and spatial resolution. J. Geophys. Res., 107, 4781, doi:10.1029/2002JD002259.

-, Y. Zhang, and W. B. Rossow, 2000: Cloud and radiation variations associated with northern midlatitude low and high sea level pressure regimes. J. Climate, 13, 312-327.

Waliser, D. E., N. E. Graham, and C. Gautier, 1993: Comparison of the highly reflective cloud and outgoing longwave radiation datasets for use in estimating tropical deep convection. J. Climate, 6, 331-353.

Williams, K. D., and G. Tselioudis, 2007: GCM intercomparison of global cloud regimes: Present-day evaluation and climate change response. Climate Dyn., 29 (2-3), 231-250, doi:10.1007/ s00382-007-0232-2.

—, and M. J. Webb, 2009: A quantitative performance assessment of cloud regimes in climate models. Climate Dyn., 33 (1), 141-157, doi:10.1007/s00382-008-0443-1.

- M. A. Ringer, and C. A. Senior, 2003: Evaluating the cloud response to climate change and current climate variability. Climate Dyn., 20, 705-721, doi:10.1007/s00382-002-0303-3.

— , and Coauthors, 2006: Evaluation of a component of the cloud response to climate change in an intercomparison of climate models. Climate Dyn., 26, 145-165, doi:10.1007/ s00382-005-0067-7.

Yang, G.-Y., and J. Slingo, 2001: The diurnal cycle in the tropics. Mon. Wea. Rev., 129, 784-801.

Zhang, Y., W. B. Rossow, A. A. Lacis, V. Oinas, and M. I. Mishchenko, 2004: Calculation of radiative fluxes from the surface to top of atmosphere based on ISCCP and other global data sets: Refinements of the radiative transfer model and the input data J. Geophys. Res., 109, D19105, doi:10.1029/2003JD004457. 